\title{
Basic Mechanisms of the Aging Gastrointestinal Tract
}

\author{
N. Salles \\ Geriatric Department, Hospital Xavier Arnozan, Pessac, France
}

\section{Key Words}

Geriatrics - Gastrointestinal aging • Motor and histological changes

\begin{abstract}
The goal of this short review is to summarize recent data on gastrointestinal changes with aging, focusing on gastrointestinal motility disorders, and mucosal variations. First of all, this review focused on gastrointestinal motility disorders with aging, even though an increased prevalence of several gastrointestinal motor disorders (i.e., dysphagia, dyspepsia, anorexia, and constipation) occurs in older people, aging per se appears to have a minor direct effect on most gastrointestinal functions. Secondly, this review focused on histological changes with aging, i.e., regulation of gastrointestinal mucosal growth, gastrointestinal carcinogenesis, and gastric mucosal changes, especially changes in gastric acid secretion, bacterial overgrowth and its consequences on elderly patients.

Copyright $\odot 2007$ S. Karger AG, Basel
\end{abstract}

\section{Introduction}

Relatively little work has been done to describe the gastrointestinal changes associated with normal aging in humans and in many instances normal data on which to base clinical comparisons are not available.
The goal of this short review is to summarize recent data on gastrointestinal changes with aging, focusing on gastrointestinal motility disorders, and mucosal variations.

\section{Aging and Gastrointestinal Motor Function}

There seems to be an increase in gastrointestinal disorders of function and motility with aging. However, even though an increased prevalence of several gastrointestinal motor disorders (i.e., dysphagia, dyspepsia, anorexia, and constipation) occurs in older people, aging per se appears to have only a minor direct effect on most gastrointestinal functions, in large part because of the functional reserve capacity of the gastrointestinal tract.

\section{Esophageal Motility}

In studying patients in their 80 and 90s, several authors have reported a significant decrease in the amplitude of peristaltic pressures, but not duration and velocity, as well as an increased frequency of non-propulsive, often repetitive contractions $[1,2]$. Morphological studies have shown an age-related loss of enteric neurons in the human esophagus. The number of neurons decreases after 70 years of age, which is accompanied by an increase in the sizes of the neurons along the esophagus being most pronounced in its superior third at the junction with the pharynx [3]. There is also evidence of reduced 
amplitude of peristaltic contractions in the lower esophagus of the elderly, consistent with the observation that esophageal clearance after gastroesophageal reflux is impaired in older individuals. Ferriolli et al. [4] confirmed that these motility abnormalities in the elderly impaired acid clearance by finding that duration of gastroesophageal reflux episodes was longer in older persons although the frequency of reflux episodes did not vary with age. Furthermore, age-related normality limits of esophageal pressures should be considered before realizing manometric explorations. In fact, results of a study performing manometric explorations in healthy persons showed that age correlated inversely with both lower and upper esophageal sphincter pressure and length, and peristaltic wave amplitude and velocity [5].

\section{Gastric Emptying and Small Intestinal Transit}

The effect of aging on gastric motility has evoked some interest, but it is still controversial whether gastric emptying changes in advanced age. Madsen et al. $[6,7]$ determined gastric emptying and small intestine transit rates in older persons (mean age $>80$ ) using a gamma camera technique. They reported no influence in gastric emptying or small intestine transit rate with advanced age, no difference in post-prandial frequency of antral contractions, and motility variables were not affected by gender or body mass index. However, other studies showed that radiolabeled liquids or digestible solids emptied more slowly from the stomach in older patients, even though the magnitude of the changes were relatively small when comparing with younger patients. Shimamoto et al. [8] enrolled active and inactive elderly subjects to investigate gastric motility using electrogastrography and the ${ }^{13} \mathrm{C}$ acetate breath test. They showed that post-prandial peristalsis and gastric contractile force were reduced in the elderly, and the reduction was greater in the inactive group than in the active one. Nakae et al. [9] determined the effect of lipid on gastric motor function in healthy elderly persons. They reported that the delaying effect of lipid on gastric emptying is increased in the elderly, and that the administration of lipase accelerates the emptying of lipid from the stomach.

Mechanisms leading to slower gastric emptying in older persons are still not defined. Research in the aging enteric nervous system (ENS) reported the phenomenon of age-related neurodegeneration of the ENS in three rodent species commonly used in enteric neurobiology (rat, mouse, guinea pig) $[10,11]$. Beyond the general observation that significant numbers of neurons in the myenteric plexus of the gastrointestinal tract are lost as these animals age, comparatively little else is known. It is unclear whether cell death affects all classes of myenteric neurons non-selectively or is confined to specific phenotypes. Even if results of different studies are still conflicting, it seems that age-related cell loss in the myenteric plexus does not occur in nitrergic neurons and that the loss occurs exclusively in the cholinergic subpopulation of enteric neurons. In a recent study, Phillips et al. [12] reported that age-related cell loss in the small and large intestines of Fischer 344 rats occurs exclusively in the cholinergic subpopulation.

\section{Colonic Transit}

It is commonly assumed that complaints of chronic constipation or alterations in colonic functioning are natural consequences of the aging process. Some studies reported a tendency towards a longer mean colonic transit time in older patients. Madsen and Graff [6] reported a slower colonic transit time in healthy persons aged $>80$. The process of normal aging seems to reduce the propulsive efficacy of the colon. Age-related changes in both the neurons and the receptors of the ENS might be one of the explanations. Morphological data from human tissues suggest that the population of colonic enteric neurons begins to decline relatively early in life, with marked decreases in both submucosal and myenteric plexuses [13]. The mechanism of delayed colonic transit time in aging remains unclear, a reduction in the number of myenteric neurons suggests that there may be a reduction in the releasable pool of neurotransmitter substances as well as fewer neurons to respond to signals. Some hypotheses are reported in the literature data. Some authors have hypothesized that advanced age was associated with decreased expression of neuronal nitric oxide synthase (NOS) and concomitant reduction in synthesis of NO in the colon. Takahashi et al. [14] reported significant reduction of NOS-immunoreactive cells and NOS synthesis in the colonic myenteric plexus of aged rats. This may be one explanation of delayed colonic transit time observed in advanced age. Other studies reported an increased density of opioid receptors in the colon of aged guinea pigs. Culpepper-Morgan et al. [15] reported that when administered orally, specific opioid antagonists were able to reverse the gastrointestinal transit delay caused by orally administered $\mu$ - and $\kappa$-opioid agonists in a dose-dependent fashion in aged guinea pigs. These data confirm the enteric action of orally administered opioids and the presence of $\kappa$-opioid activity in the guinea pig ENS. Accordingly, specific opioid antagonists appeared to improve chronic constipation in geriatric pa- 
tients. Other studies reported reduced release of acetylcholine in response to electrical field stimulation in old rat colonic preparations, with a decrease by $50 \%$ with age. Roberts et al. [16] reported age-dependent reduction in acetylcholine release in the rat colon myenteric plexus that may, in part, be associated with decreased calcium influx via membrane calcium channels. Furthermore, Powell and Reddix [17] reported that colonic tissues from older animals diminished secretory responses to nicotinic agonists when comparing with the preparations from younger animals.

\section{Aging and Gastrointestinal Histological Changes}

\section{Regulation of Gastrointestinal Mucosal Growth}

The gastrointestinal tract represents an organ system that is characterized by rapid proliferation, aging gastrointestinal tissues illustrate markedly different phenomena from aged post-mitotic cells. A state of hyperproliferation occurs in the epithelial cells of the stomach, the small intestine, and the large intestine of stable-fed, aged rodents when compared to young mature rodents [18]. In fact, the number of gastric and colonic mucosal cell undergoing apoptosis was found to be lower in older animals [19]. Furthermore, abnormalities of proliferative and differentiation responses become evident when gastrointestinal tissues are stimulated by injury, or by starvation and refeeding. Gastrointestinal mucosal cell proliferation is known to be under the regulation of nutritional factors. Holt et al. [20] demonstrated that a restriction of calories in aging rats was associated with a significantly higher apoptotic index in the jejunum and colon. These observations suggest that nutritional modulation of mucosal cell proliferation is affected by aging. Age-associated changes in gastrointestinal mucosal cell proliferation could also be secondary to alterations in hormonal influences, especially in the gastric mucosa. Majumdar [21] reported that responsiveness of the gastric mucosa to different peptides, i.e., gastrin, bombesin, epidermal growth factor, changes at different stages of life. For example, some results demonstrated an age-related decline in gastrin secretion that could partly be attributed to a higher ratio of somatostatin to gastrin cells in the antral mucosa. There is also a progressive loss of gastric mucosal responsiveness to both acid secretory and growth-promoting actions of gastrin. One explanation could be the loss of functional receptors of gastrin with age.

\section{Aging and Gastrointestinal Carcinogenesis}

One of the most consistent pathological observations in senescent animals is the increased incidence of many types of malignancies, including gastric and colorectal cancers. In elderly patients the incidence of digestive cancers increases subsequently with peak incidence occurring in the seventh decade. Many reasons including altered carcinogen metabolism and long-term exposure of cancer-causing agents have been offered for the age-dependent rise in malignancies. Carcinogenesis results from the accumulation of mutations during progression from normal epithelium to carcinoma. For colon cancer, studies reported that the loss or inactivation of the tumor suppressor gene APC (adenomatous polyposis coli) initiated genomic instability which produced phenotypic appearance of an adenoma. With the inactivation of the tumor suppressor APC, additional alterations in tumor suppressor (p53, DCC) and oncogenes may accumulate and lead to the development of an adenomatous polyp and eventually to a carcinoma [22]. Recently, Majumdar [21] reported a higher incidence of mutations of several tumor suppressor genes, specifically APC, DCC and p53 in the gastric mucosa of older patients.

\section{Gastric Changes with Aging}

Between 1920 and 1980, many studies reported a significant reduction in gastric acid secretion with age. The majority of these studies were retrospective and did not take into account the presence of possible gastric atrophic lesions. More recent studies including old patients $>80$ without gastric atrophic lesions showed that gastric acid secretion remained normal with age, $90 \%$ of the patients had normal acid secretion [23]. Haruma et al. [24] reported that advancing age had no influence on gastric acid secretion in Helicobacter pylori-negative patients, while it decreased with age in $H$. pylori-positive patients. The decline in gastric acid secretion in H. pylori-positive patients depended on both increasing prevalence of fundic atrophic gastritis and inflammatory cytokines, i.e., IL-1 $\beta$ and TNF- $\alpha$, which are known to inhibit parietal cells. Epidemiological studies reported an increase prevalence of atrophic gastritis in elderly patients, with rates ranging between 50 and $70 \%$ in patients $>80$ [25]. A series of studies, mainly from Japan, has focused on the longterm effects of $H$. pylori infection and its role in the development of the histological changes that occur with aging, i.e., atrophic gastritis [25]. In a large multicenter trial, authors reported that both atrophic gastritis and intestinal metaplasia were strongly associated with $H$. $p y$ lori infection and not with aging per se [26]. 
As a result of atrophic gastritis, there is decreased acid secretion which may lead to two consequences in the elderly: bacterial overgrowth in the proximal intestinal tract and gastrointestinal malabsorption. Parlesak et al. [27] performed a study to determine the prevalence of small bowel bacterial overgrowth in older adults using a hydrogen breath test. They also tried to assess whether it was associated with abdominal complaints and nutrient intake. Their results showed a $15.6 \%$ prevalence of small bowel bacterial overgrowth. They showed that intake of inhibitors of gastric acid production contributed significantly to the high prevalence of a positive breath test in older adults, which was associated with lower body weight, lower body mass index, lower plasma albumin concentration, and higher prevalence of diarrhea. In geriatrics, malnutrition is one of the clinical consequences of bacterial overgrowth, and antibiotic treatment may lead to improve anthropometric parameters of these patients [28].

Gastric malabsorption may be another consequence of reduced acid gastric secretion. In fact, malabsorption of food-bound cobalamin may be due to reduced gastric acid production in elderly patients, combined with bacterial overgrowth. Decreased gastric acid production may lead to reduced release of free vitamin $\mathrm{B}_{12}$ from food protein [29]. Also, hypochlorhydria causes intestinal bacterial overgrowth, which interferes with vitamin $B_{12}$ absorption. There are contradictory data in the literature on the effect of atrophic gastritis on vitamin $B_{12}$ status in the elderly. Van Asselt et al. [29] found no significant difference in vitamin $B_{12}$ absorption (free or protein-bound) between subjects $<64$ years and those $\geq 65$ years (median 75 years). In contrast, Scarlet et al. [30] demonstrated that a reduction with age in dietary vitamin $\mathrm{B}_{12}$ absorption was related to elevated serum gastrin levels, which indicates hypochlorhydria. Kaptan et al. [31] reported in 77 $H$. pylori-positive patients with low serum vitamin $B_{12}$ levels a normalization of cobalamin levels in $40 \%$ after $H$. pylori eradication. Moreover, previous studies have demonstrated that treatment of patients with a regimen of antibiotics, including tetracycline, can increase food-cobalamin absorption and correct low serum vitamin $\mathrm{B}_{12}$ levels. Atrophic gastritis may also affect calcium ferric iron and calcium absorption. Both calcium and ferric iron are kept soluble, and hence, absorbable in the intestinal milieu through the acidifying effects of gastric acid.

In summary, atrophic gastritis is strongly associated with chronic $H$. pylori infection, and this may induce hypochlorhydria with bacterial overgrowth and malabsorp- tion. Chronic infection may also lead to chronic gastritis with chronic inflammation in the gastric mucosa. This may have several consequences such as an increased production of reactive oxygen species (ROS) [32]. There is increasing evidence that ROS play a key role in the processes of tissue damage and aging [33]. In the stomach mucosa, ROS might directly damage or kill epithelial cells, and thereby contribute to atrophy. However, more complex mechanisms of action are conceivable. ROS may lead to a heightened sensitivity of the aging stomach to proinflammatory stimuli, and may damage DNA, thereby leading to mutations and cancerogenesis. The molecular sources of ROS that contribute to the aging process are still a matter of debate. ROS generation by mitochondria has been consistently implied, but more recently the role of the NOX family of superoxide-generating NADPH oxidases is receiving increased attention. Previous studies on expression of NADPH oxidases in gastric mucosa have mainly been performed in guinea pigs. In this system, NOX1 (an NADPH oxidase with its main localization in the colon) was found and proposed to be involved in the regulation of the inflammatory response and/or cell proliferation [34].

Chronic inflammation in the gastric mucosa may also affect expression of gastric satiety inducible peptides such as leptin or ghrelin, which may play a role in the regulation of food intake. Recent evidence supports the view that in humans and rats, leptin is secreted not only from adipose tissues but also from the gut [35]. Studies reported that gastric inflammation induced by $H$. pylori infection raised gastric leptin expression which induced satiety and lower body mass index [36]. Ghrelin is a recently discovered peptide which is produced mainly in the stomach, and which has been implicated in the control of food intake and energy homeostasis in both humans and rodents. A recent study, authors reported that cure of $H$. pylori infection increased plasma ghrelin, which in turn may lead to increased appetite and weight gain [37]. Chronic gastric inflammation may, thus, induce variations of expression of both leptin and ghrelin and may play a role in the physiopathology of anorexia in elderly patients.

\section{Small Bowel Changes with Aging}

Conflicting reports have been published regarding intestinal mucosa. While studies in rodents have suggested an age-related loss of both villous and enterocyte height, most studies in humans have not shown any age-related changes in the small intestinal architecture. No changes were observed when looking at the surface areas of the small intestine, crypt depth, villous height, crypt-to-vil- 
lus ratio, enterocytes, brush border, and Brunner glands [38].

Age-related changes in intestinal absorption have been studied. Authors reported a decreased absorption of Dxylose in aging humans. This may be explained by two mechanisms: poor renal function and the presence of bacterial overgrowth [39]. In fact, D-xylose excretion is dependent on renal function, frequently impaired with aging. When excluding patients with impaired renal function, results show only a modest reduction in xylose absorption associated with aging. In addition, bacterial overgrowth of the small intestine may induce falsely positive breath tests results. Anaerobic bacteria can produce proteases that reduce carbohydrate absorption. When regarding lipid absorption, results are contradictory [40]. In animals, results show a reduced gastric lipase and bile acid secretion, decreasing lipid solubilization and thus decreasing lipid absorption. In humans, a study of only healthy aged showed no correlation between age and $72 \mathrm{~h}$ fecal fat excretion. Other studies in humans reported that absorption of fat may take longer in the elderly, and that post-prandial serum bile acid levels may be reduced with aging. The prolonged absorption of fats in elderly may induce post-prandial satiety, reducing overall intake in the elderly.

Studies in animals reported an impaired adaptive response of the aged intestine. In time of stress, like dietary restriction initiated in aged rats, results show dramatic weight loss without stabilization after reintroduction of normal diet. The intestine of the animals was atrophied and ileal hydrolase activity was decreased. Following a period of stress, like illness or injury, it has been shown that elderly patients continued to underfeed themselves for 10-15 days while younger patients increased their energy intake [39]. Elderly patients may have decreased functional reserve of the intestine and may become undernourished more rapidly during acute hospitalizations, and may require an extended period of intensive nutritional monitoring because of reduced adaptive responses.

\section{References}

$>1$ Ferriolli E, Dantas RO, Oliveira RB, et al: The influence of aging on oesophageal motility after ingestion of liquids with different viscosities. Eur J Gastroenterol Hepatol 1996;8:793-798.

$>2$ Tack J, Vantrappen G: The aging esophagus. Gut 1997;41:422-424.

$\checkmark 3$ Meciano Filho J, Carvalho VC, de Souza RR: Nerve cell loss in the myenteric plexus of the human esophagus in relation to age: a preliminary investigation. Gerontology 1995; 41:18-21.

$\checkmark 4$ Ferriolli E, Oliveira RB, Matsuda NM, et al: Aging, esophageal motility and gastroesophageal reflux disease. J Am Geriatr Soc 1998; 46:1534-1537.

5 Grande L, Lacima G, Ros E, et al: Deterioration of esophageal motility with age: a manometric study of 79 healthy subjects. Am J Gastroenterol 1999;94:1795-1801.

$\checkmark 6$ Madsen JL, Graff J: Effects of aging on gastrointestinal motor function. Age Aging 2004;33:154-159.

7 Madsen JL: Effects of gender, age, and body mass index on gastrointestinal transit times. Dig Dis Sci 1992;37:1548-1553.

$>8$ Shimamoto C, Hirata I, Hiraike Y Evaluation of gastric motor activity in the elderly by electrogastrography and the ${ }^{13} \mathrm{C}$-acetate breath test. Gerontology 2002;48:381-386.

-9 Nakae Y, Onouchi H, Kagaya M, et al: Effects of aging and gastric lipolysis on gastric emptying of lipid in liquid meal. J Gastroenterol 1999;34:445-449.
10 Phillips RJ, Powley TL: As the gut ages: timetables for aging of innervation vary by organ in the Fisher 344 rat. J Comp Neurol 2001; 434:358-377.

11 El-Salhy M, Sandstrom O, Holmlund F: Ageinduced changes in the enteric nervous system in the mouse. Mech Aging Dev 1999;107: 93-103.

12 Phillips RJ, Kieffer EJ, Powley TL: Aging of the myenteric plexus: neuronal loss is specific to cholinergic neurons. Auton Neurosci 2003;106:69-83.

13 Gomes OA, de Souza RR, Liberti EA: A preliminary investigation of the effects of aging on the nerve cell number in the myenteric ganglia of the human colon. Gerontology 1997;43:210-217.

14 Takahashi T, Qoubaitary A, Owyang C, et al: Decreased expression of nitric oxide synthase in the colonic myenteric plexus of aged rats. Brain Res 2000;883:15-21.

15 Culpepper-Morgan JA, Holt PR, LaRoche D, et al: Orally administered opioid antagonists reverse both $\mu$-and $\kappa$-opioid agonist delay of gastrointestinal transit in the guinea pig Life Sci 1995;56:1187-1192.

16 Roberts D, Gelperin D, Wiley JW: Evidence for age-associated reduction in acetylcholine release and smooth muscle response in the rat colon. Am J Physiol 1994;267:G515G522.

17 Powell AR, Reddix RA: Differential effects of maturation on nicotinic- and muscarinic receptor-induced ion secretion in guinea pig distal colon. Proc Soc Exp Biol Med 2000; 224:147-151.

18 Atillasoy E, Holt PR: Gastrointestinal proliferation and aging. J Gerontol 1993;48:B43B49.

19 Xiao ZQ, Moragoda L, Jaszewski R, et al: Aging is associated with increased proliferation and decreased apoptosis in the colonic mucosa. Mech Aging Dev 2001;122:1849-1864.

20 Holt PR, Moss SF, Heydari AR, Richardson A: Diet restriction increases apoptosis in the gut of aging rats. J Gerontol A Biol Sci Med Sci 1998;53:B168-B172.

21 Majumdar AP: Regulation of gastrointestinal mucosal growth during aging. J Physiol Pharmacol 2003;54:S4:143-154.

-22 Fearon ER, Vogelstein B: A genetic model for colorectal tumorigenesis. Cell 1990;61:759767.

23 Hurvitz A, Brady DA, Schaal E, et al: Gastric acidity in older adults. JAMA 1997;278:659662.

24 Haruma K, Kamada T, Kawaguchi H, et al: Effect of age and Helicobacter pylori infection on gastric acid secretion. J Gastroenterol Hepatol 2000;15:277-283.

25 Pilotto A, Salles N: Helicobacter pylori infection in geriatrics. Helicobacter 2002;7:5662.

26 Asaka M, Sugiyama T, Nobuta A, et al: Atrophic gastritis and intestinal metaplasia in Japan: results of a large multicenter study. Helicobacter 2001;6:294-299. 
27 Parlesak A, Klein B, Schecher K, et al: Prevalence of small bowel bacterial overgrowth and its association with nutrition intake in nonhospitalized older adults. J Am Geriatr Soc 2003;51:768-773.

28 Lewis SJ, Potts LF, Malhotra R, et al: Small bowel bacterial overgrowth in subjects living in residential care homes. Age Aging 1999; 28:181-185.

29 Van Asselt DZ, van der Broek WJ, Lamers $\mathrm{CB}$, Corstens FH, Hoefnagels WH: Free and protein-bound cobalamin absorption in healthy middle-aged and older subjects. J Am Geriatr Soc 1996;44:949-953.

>30 Scarlet JD, Read H, O’Dea K: Protein-bound cobalamin absorption declines in the elderly. Am J Hematol 1992;39:79-83.
31 Kaptan K, Beyan C, Ural A, et al: Helicobacter pylori: is it a novel causative agent in vitamin $\mathrm{B}_{12}$ deficiency? Arch Intern Med 2000;160:1349-1353.

32 Pignatelli B, Bancel B, Plummer M, et al: $\mathrm{He}$ licobacter pylori eradication attenuates oxidative stress in human gastric mucosa. Am Gastroenterol 2001;96:1758-1766.

33 Lenaz G, Bovina C, D’Aurelio M, et al: Role of mitochondria in oxidative stress and aging. Ann NY Acad Sci 2002 959:199-213.

34 Teshima S, Kutsumi H, Kawahara T, et al: Regulation of growth and apoptosis of cultured guinea pig gastric mucosal cells by mitogenic oxidase 1. Am J Physiol 2000;279: G1169-G1176.

35 Bado A, Levasseur S, Attoub S, et al: The stomach is a source of leptin. Nature 1998; 394:790-793.
36 Azuma T, Suto H, Ito Y, et al: Gastric leptin and Helicobacter pylori infection. Gut 2001; 49:324-329.

37 Nwokolo CU, Freshwater DA, O’Hare P, et al: Plasma ghrelin following cure of Helicobacter pylori. Gut 2003;52:637-640.

38 Corazza GR, Frazzoni M, Gatto MR, et al: Aging and small-bowel mucosa: a morphometric study. Gerontology 1986;32:60-65.

39 Woudstra T, Thomson ABR: Nutrient absorption and intestinal adaptation with aging. Best Pract Res Clin Gastroenterol 2002; 16:1-15.

40 Holt PR, Balint JA: Effects of aging on intestinal lipid absorption. Am J Physiol 1993; 264:G1-G6. 